\title{
Calibration of the power-recycled gravitational wave detector, GEO 600
}

\author{
M. Hewitson ${ }^{\text {a) }}$ \\ Physics and Astronomy, University of Glasgow, Glasgow G12 8QQ, Great Britain \\ H. Grote and G. Heinzel \\ Max-Planck-Institut für Gravitationsphysik, Albert-Einstein-Institut, Aussenstelle Hannover, Callinstrasse 38, \\ 30167 Hannover, Germany \\ K. A. Strain and H. Ward \\ Physics and Astronomy, University of Glasgow, Glasgow G12 8QQ, Great Britain \\ U. Weiland \\ Institut für Atom- und Molekülphysik, Universität Hannover, Callinstrasse 38, 30167 Hannover, Germany
}

(Received 1 April 2003; accepted 6 May 2003)

GEO 600 is an interferometric gravitational wave detector which, when completed, will employ a novel, dual-recycled optical scheme allowing its optical response to be tuned over a range of frequencies. The accurate calibration of the sensitivity of GEO to gravitational waves is a critical part of preparing GEO data for exchange with other detectors worldwide and for the extraction of scientific results. The final optical scheme that will be used in GEO will make it necessary to combine more than one output signal to optimally recover a calibrated strain channel. An on-line calibration scheme has been developed with a view to covering both the power-recycled and dual-recycled interferometer configurations of GEO. The recent worldwide coincident science run (S1) proved an excellent opportunity to test the calibration scheme of GEO in a power-recycled mode. The ability to track and study the stability of the calibration over long time scales was invaluable. A number of areas have been highlighted where improvement is necessary, but the scheme proved successful and produced a continuous real-time $h(t)$ for the entire duration of the S1 science run. Consideration of the errors discussed throughout this article lead to a calibration accuracy for this science run of around $4 \%$ at high frequencies $(>200 \mathrm{~Hz})$ and about $6 \%$ for frequencies between about 50 and $200 \mathrm{~Hz}$. This article presents the on-line calibration scheme that was developed for, and employed during, the recent S1 science run. The hardware signal injection is presented in detail followed by the on-line analysis method that allows real-time calibration of this interferometer configuration. The results of the calibration process are discussed and attention is drawn to the successes and to the points where improvement can be made. (C) 2003 American Institute of Physics. [DOI: 10.1063/1.1597959]

\section{INTRODUCTION}

GEO 600 is a gravitational wave observatory near Hannover, Germany, and is a collaboration of UK and German institutions. Currently the detector is undergoing the final stages of hardware installation and will soon enter a phase of continuous operation. When operational, GEO 600 should be one of the most sensitive displacement measuring instruments in the world, capable of measuring differential displacements between the two $1200 \mathrm{~m}$ long arms of the order $10^{-19} \mathrm{~m} / \sqrt{\mathrm{Hz}}$ in the frequency range $50 \mathrm{~Hz}$ to a few $\mathrm{kHz}$.

The design of GEO 600 is based on the well known Michelson interferometer but with the addition of two mirrors: ${ }^{1}$ the power recycling (PR) mirror which will increase the circulating power in the detector, lowering the shot-noise limit, and the signal recycling (SR) mirror which forms a resonant cavity capable of enhancing signals in a chosen frequency band. Another noticeable difference of GEO 600 from a basic Michelson interferometer is that the

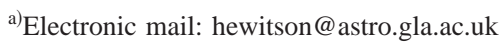

two arms are folded (Fig. 1). For the S1 science run, GEO was in an intermediate design configuration with only the power-recycling system in place.

Astrophysical signals are typically sought in four categories: from the inspiral of compact objects (neutron stars, black holes), from sources producing periodic waves (e.g., pulsars), from the stochastic gravitational wave (GW) background, and from bursts of short duration (a few cycles of the dominant signal frequency). An accurate calibration of the detector is necessary to interpret these astrophysical signals and, in the case when no individual signals can be identified, to set limits on their possible magnitudes. In the powerrecycled configuration of GEO, all the gravitational wave information is contained in a single output channel so it is possible to perform astronomical searches on the uncalibrated detector output and then calibrate the results. When dual-recycling is installed this will no longer be the case. Multiple signals will need to be combined to produce a calibrated strain channel $[h(t)]$ with optimal signal-to-noise ratio. To overcome this difficulty, it was decided within the GEO collaboration that effort would be focused on produc- 


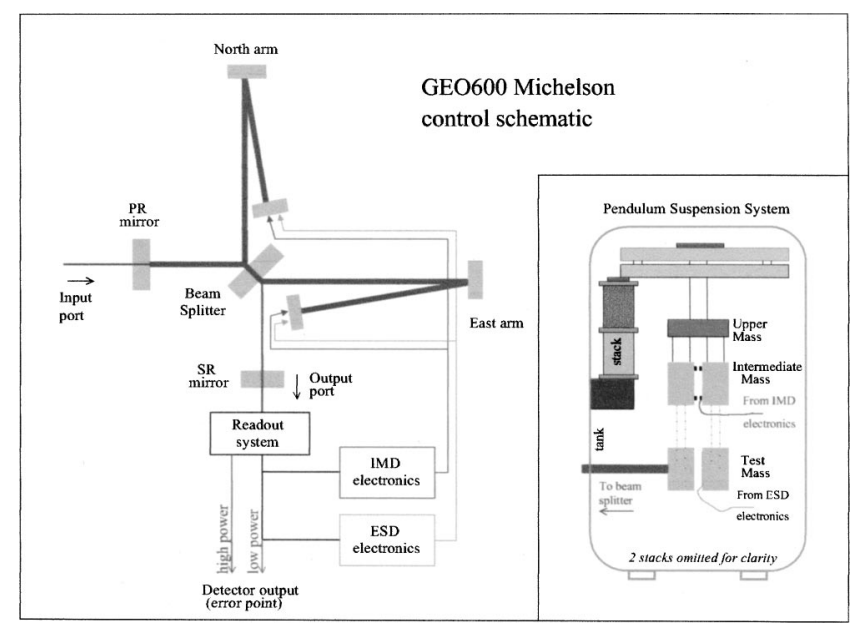

FIG. 1. A schematic of the Michelson lock for GEO 600. The electrostatic drive (ESD) applies high frequency correction forces to the mirror. The intermediate mass drive (IMD) applies low frequency corrections to the intermediate mass. In reality the arms are folded in a vertical plane, and are shown here in horizontal plane for convenience.

ing calibrated data streams that could be presented to the different analysis pipelines rather than providing information with which to calibrate any analysis results. The uncalibrated data, together with sufficient information to reproduce the calibration, will be also archived. The production of a calibrated detector output also provides a clear point of control over the quality and accuracy of the $h(t)$ signal. As will be explained below, calibration here means not only the determination of a fixed factor, but more generally the reconstruction in the time-domain of an underlying detector stimulus (mirror displacement or gravitational wave strain) from several channels that have different and possibly varying frequency dependencies and signal-to-noise ratios.

During the summer of 2002 GEO 600 took part in a coincident science run (S1) with other detectors worldwide. One of the main science goals of the run was to set upper limits on the GW emission from a variety of astronomical sources. For this run GEO was operated as a power-recycled Michelson interferometer; work on the dual-recycled interferometer started after S1.

\section{THE MICHELSON LOCKING SCHEME}

GEO relies on a complex control system with many feedback paths. To calibrate the power-recycled Michelson interferometer we need only consider the feedback loop that controls the lock of the Michelson. The Michelson feedback loop senses, and minimizes, deviations from the optimal dark fringe operating point. In order to obtain the required loop bandwidth necessary to optimize noise performance and dynamic range, a split feedback servo is adopted (Fig. 1). Low frequency feedback signals are applied with a coil and magnet to an intermediate mass drive (IMD) from which the main (test mass) mirror is suspended. High frequency feedback signals are applied directly to the test mass via an electrostatic drive. In each case, the forces are applied from a secondary pendulum chain suspended behind the main pendulum chain. ${ }^{2}$ Due to the presence of violin mode resonances of the suspensions, there is a limit to the amount of loop gain that can be achieved. The limited bandwidth of the servo means that we need to record the error-point signal in order to recover signal information at frequencies out with the servo bandwidth. Although for the S1 science run, the detector noise was sufficiently high that it was possible to measure the signal at all frequencies in the detection band with high signal-to-noise ratio, this may not be the case in the future, particularly when signal-recycling is installed. In that case we may need to recover the signal from the feedback signal for frequencies within the bandwidth of the servo in order to maintain a high enough signal-to-noise ratio during the measurement process. For S1, measuring the size of the Michelson error-point signal and determining the characteristics of all the elements of the servo loop provides all the information necessary to determine the mirror motion that would be present without the action of the servo in the detection band. From the recovered displacement signal, we can then calculate the strain, $h(t)$. Observations suggest that the calibration of the feedback actuators remains constant over time. However, the presence of tidal drifts, temperature fluctuations, and seismic disturbances can all cause changes in the alignment of the optics and these misalignments cause the sensitivity of the detector to vary in a time dependent way. The overall calibration must therefore be determined on time scales much shorter than any of these drift times.

During the S1 science run, two photodiodes were used to sense the output light of the interferometer: a low-power quadrant photodiode used in the Michelson servo-loop and the auto-alignment system, and a high-power photodiode to give a high signal-to-noise ratio measure of the output light. It was from the high-power photodiode that the differential mirror displacement (and ultimately the detected strain) was derived. Because this photodiode is not part of the Michelson servo loop, it was necessary to determine the relative gain between it and the in-loop photodiode denoted $H_{\text {rel }}$ in Fig. 4. Both photodiode outputs were passed through whitening filters and recorded by the data acquisition system.

\section{CALIBRATION OF GEO 600}

An optimally oriented gravitational wave of amplitude $h(t)$ passing through GEO will give rise to a length change, $\Delta L$, in each arm given by

$$
h(t)=2 \frac{\Delta L(t)}{L},
$$

where $L$ is $1200 \mathrm{~m}$ for GEO. Calibration can be viewed as the determination of $\Delta L$ from the detector output, i.e., the determination of the detector gain, as a function of frequency, in volts per meter. We can consider the calibration in two regimes: a high frequency regime where the Michelson control servo no longer acts $(>200 \mathrm{~Hz})$ and a low frequency regime where the loop gain of the servo is significant. For both regimes the optical response of the power-recycled Michelson is flat up to $\sim 100 \mathrm{kHz}$. At frequencies well above the unity gain point of the Michelson servo the optical gain can be determined simply by displacing the end mirrors a 


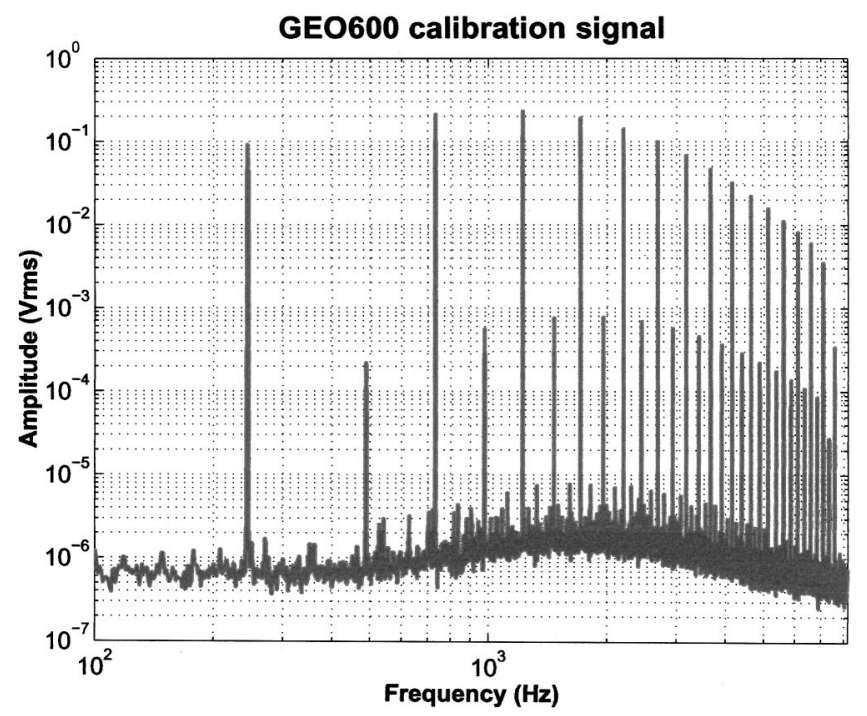

FIG. 2. Spectrum of the calibration signal applied to the ESD. The transfer function of the actuator, from applied-volts to induced-displacement, has a $1 / f^{2}$ frequency response above the pendulum resonance (which is around 1 $\mathrm{Hz})$.

known amount and then observing the detector output. For the low frequency gain, the loop-gain of the Michelson servo must also be determined.

\section{A. Calibration signal injection}

For S1, mirror motion was induced continuously at particular frequencies by injecting a calibration signal into the electrostatic drive feedback path. The signal was comprised of a forest of spectral peaks produced by differentiating a square wave oscillator such that the induced displacements from the first four odd harmonics were approximately uniform. Higher frequencies were suppressed to avoid saturation of the feedback actuator. As with the data acquisition system (DAQ) sampling clock, the oscillator was locked to a GPS frequency standard to ensure that power from any particular peak would be confined to a single frequency bin in the analysis. Figure 2 shows the injected signal which was recorded continuously throughout S1. Figure 3 shows the calibration lines as they appear in a snapshot of the detector error-point signal. A fundamental frequency of $244 \mathrm{~Hz}$ was chosen so that the calibration lines did not overlap with any other lines present in the error-point spectrum and because there was relatively little loop gain above $200 \mathrm{~Hz}$.

\section{B. Measuring the optical gain}

We estimated the optical gain, $H_{1}$ (see Fig. 4), of the detector, once per second, making the assumption that it is a slowly varying parameter. At each calibration peak, the optical gain is given by

$$
H_{1_{f}}=\frac{\left|y_{f}\right|}{\left|x_{f}\right| H_{5_{f}}}
$$

where $y_{f}$ is the calibration peak amplitude measured in the error-point spectrum, $x_{f}$ is the calibration peak amplitude applied to the electrostatic drives, and $H_{5_{f}}$ is the electrostatic

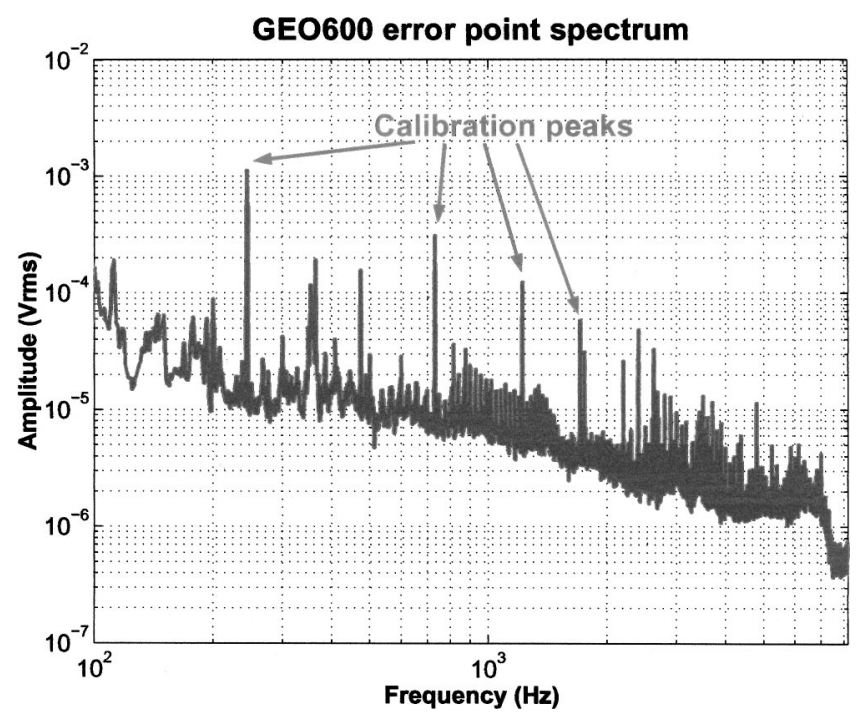

FIG. 3. A Michelson error-point spectrum from GEO taken by averaging ten $1 \mathrm{~s}$ data segments.

drive response (as seen in Fig. 4). The subscript $f$ denotes evaluation at a particular frequency. Each component of Eq. (2) was evaluated/measured at 732, 1220, and $1708 \mathrm{~Hz}$. Since these three calibration lines are well above the unity gain frequency of the Michelson servo, and appear with large signal-to-noise ratios in the error-point (SNR 50), they each provide independent estimates of the optical gain. The three estimates were combined (each weighted by their individual signal-to-noise ratios) to give a single value for the optical gain. We smoothed these $1 \mathrm{~Hz}$ estimates further using a Hanning low-pass filter of length $60 \mathrm{~s}$.

In addition, the calibration peaks were measured in the low-power photodiode and the ratio between the high- and low-power measurements was taken to get an estimate of the relative gain between the two photodiodes. Again a weighted average was made of the three estimates and a $60 \mathrm{~s}$ Hanning low-pass filter used to smooth the resulting measurements.

The two estimated gains (optical and relative), together with their variances, were recorded to disk in the main detector data stream. In addition, the low-pass filtered gains were also recorded. This provides a continuous record of the calibration scheme performance for the entire S1 run.

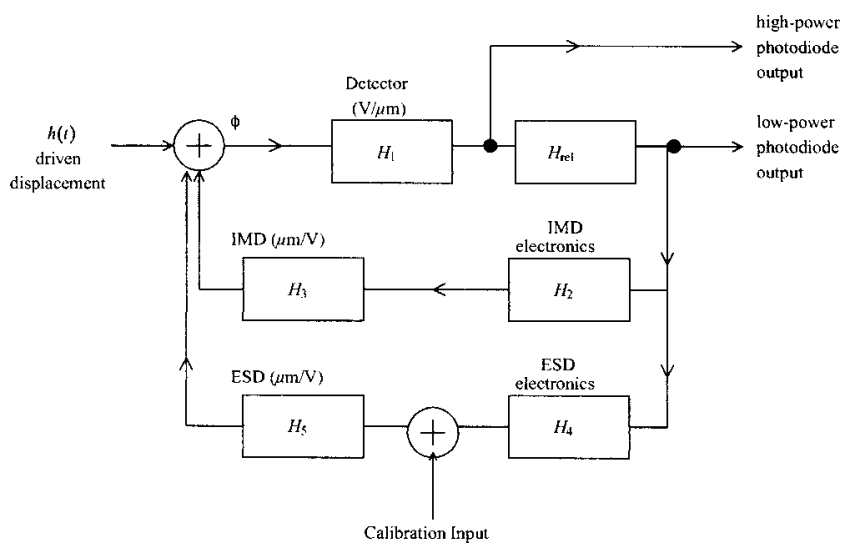

FIG. 4. A model of the Michelson lock of GEO showing the two photodiodes and the two feedback paths. 


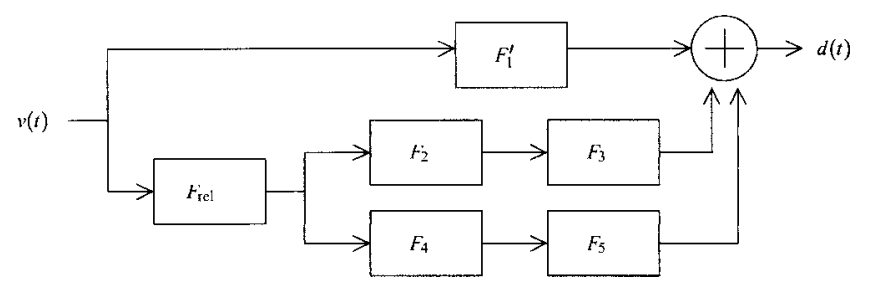

FIG. 5. The system diagram of the calibration filters.

\section{A detector model}

Having determined the calibration factor for high frequencies (the optical gain), we can then calculate the low frequency calibration factor as a function of frequency. This is best achieved using a model of the locking servo. A suitable model is shown schematically in Fig. 4 in which the gain of the optical cavity is denoted by $H_{1}$, the relative gain between the two photodiodes by $H_{\text {rel }}$, and the two feedback paths by $\mathrm{H}_{2} \mathrm{H}_{3}$ and $\mathrm{H}_{4} \mathrm{H}_{5}$. The injection point for the calibration signal is also shown. Determination of all the elements of this model (those shown as boxes in Fig. 4) allows the loop gain to be calculated at all frequencies where the model is valid. The complex closed loop transfer function of the system from detector output (in volts) to mirror displacement (in meters) can be expressed as

$$
C(j \omega)=\frac{H_{1}(j \omega)}{1+G(j \omega)},
$$

where the open loop gain, $G(j \omega)$, is given by

$$
G=H_{1} H_{\text {rel }}\left[H_{2} H_{3}+H_{4} H_{5}\right] .
$$

For this configuration of GEO, the only dynamic parts of the servo loop are the detector (or optical) gain, $H_{1}$, and the relative gain, $H_{\text {rel }}$. All other elements can be fixed and were determined prior to the run. We assumed here that the calibration of the electrostatic drives (ESDs) was not changing over time. This assumption was later validated by comparing calibrations of the ESDs from before and after the science run.

\section{Real-time calibration software}

To calibrate the detector in real time, the calibration scheme was implemented in the time domain. This allowed us to use fast infinite impulse response (IIR) filters. Using the transfer function [Eq. (3)] we can derive a time domain expression using IIR filters, $F_{\text {rel }}, F_{1} \cdots F_{5}$, in place of the frequency domain transfer functions, $H_{\text {rel }}, H_{1} \cdots H_{5}$. Thus for a recorded detector output, $v(t)$, we can determine the time varying mirror displacement, $d(t)$, by applying these filters in the appropriate combination. The implementation of this is shown as the time-domain system diagram in Fig. 5. The primed filter $F_{1}^{\prime}$ has the same frequency response as $1 / H_{1}$, in this case, a flat response. The following equation shows the system diagram as an analytic expression:

$$
d(t)=v(t) *\left\{F_{1}^{\prime}+F_{\text {rel }} *\left(F_{2} * F_{3}+F_{4} * F_{5}\right)\right\},
$$

where the asterisk denotes convolution.

Since the optical response, $H_{1}$, is flat in frequency, the filter $F_{1}^{\prime}$ is simply a time-varying gain (the inverse of the optical gain, $H_{1}$ ). The transfer function between the two photodiodes is also flat, so the filter $F_{\text {rel }}$ is also just a timevarying gain. Filters $F_{2} \cdots F_{5}$ were designed using LISO $^{3}$ to match measured transfer functions for the servo electronics and the modeled transfer functions of the feedback actuators. Since the detector operators may wish to optimize performance by varying the electronic gains in the feedback loops, these gains are held in a configuration file which is checked periodically for updates. Changing the gain of an IIR filter is simple and requires no redesigning of the filter; the denominator coefficients can simply be renormalized to give the required gain. ${ }^{4}$ Although the frequency responses of the feedback actuators are well modeled, the dc gain factors needed to be measured.

The necessary steps (not necessarily in the order of application) to calibrate the recorded error-point are: dewhiten the recorded error-point; convert the signal from volts to displacement using the estimated optical gain; correct for the loop-gain of the Michelson servo using the detector model; and convert from displacement to strain.

To avoid time-domain discontinuities that would arise if we simply updated the optical gain, $F_{1}$, once per second, the measured optical gain values were resampled to the sampling frequency $(16384 \mathrm{~Hz})$ of the detector data acquisition system using a spline interpolation algorithm. The result is that each sample of the recorded error point can be multiplied by a smoothly changing gain at the same sample rate. Using the same method, and for the same reason, the relative gain was also upsampled from 1 to $16384 \mathrm{~Hz}$. We assumed here that the gains are varying smoothly on time scales slower than 2 $\mathrm{s}$ so that no aliasing occurs because of the $1 \mathrm{~Hz}$ sampling. As can be seen in Sec. IV, this assumption turned out to be invalid since some amplitude modulation of the calibration lines can still be seen after the calibration process.

Since the signal is stored as double precision floating point numbers which have a dynamic range of about $10^{15}$, it is necessary to keep the dynamic range of the signal well within this range. We decided to try to keep the dynamic range of calibrated strain signal within seven orders of magnitude. Since the detection band of GEO is defined as being between $50 \mathrm{~Hz}$ and a few $\mathrm{kHz}$, it was possible to suppress the large low-frequency signal due to seismic noise by highpass filtering the signal above $10 \mathrm{~Hz}$. This was done before the signal was dewhitened and before the calibration filters were applied (both processes amplify the low-frequency components). To restrict the dynamic range further, it was desirable to suppress the calibration lines as much as possible. Using the model filter for the ESD, it is possible to simulate the error-point of the Michelson interferometer control servo for the particular frequencies of the calibration lines. The simulated error-point can then be added (or subtracted, depending on the phase) to the recorded error-point signal. Each calibration line is then suppressed as much as its signal-to-noise ratio allows. Figure 2 shows the noise of the calibration signal to be five orders of magnitude below the calibration peaks. Since the peaks appear in the error-point spectrum with signal-to-noise ratios of between 10 and 50, the noise added to the recorded error-point during this process is negligible. 


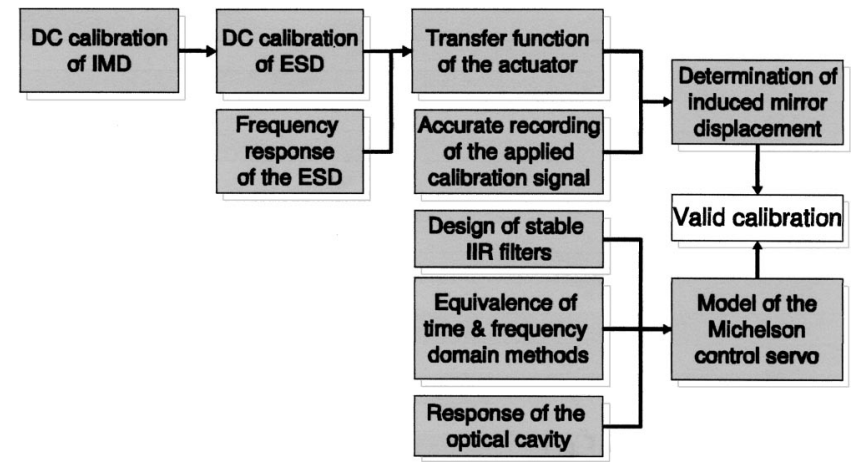

FIG. 6. A sketch of the chain of dependency that leads to a valid calibration.

\section{E. The sign of GEO 600}

The sign of the derived $h(t)$ signal for S1 was determined before the run began and was based on the definition that an increase in the North arm length is considered as a positive increase in strain. By tracing the phase of the applied calibration signals through both the Michelson control subsystem and through the data acquisition system, we deduced that there is $180^{\circ}$ phase offset between the applied calibration signal (and hence any gravitational wave signal), and the derived $h(t)$ signal.

\section{RESULTS AND VALIDATION}

Validating the calibration scheme is best done in a hierarchical way. Figure 6 shows the chain of logic that was used to validate the calibration process.

Starting at the upper-left corner of Fig. 6, the intermediate mass actuator was calibrated by fringe counting. Here we drive the IMD longitudinally with a low frequency signal (around $100 \mathrm{mHz}$ ) and observe the fringes measured by the output photodiode with the detector unlocked. By counting the number of fringes between turning points of the pendulum swing, we can determine the number of fringes we get per volt applied. Using the fact that one fringe corresponds to $0.532 \mu \mathrm{m}$ displacement of the mirror $(\lambda / 2)$, we get the calibration of IMD to be $\sim 100 \mu \mathrm{m} / \mathrm{V}$. The measurement was repeated many times and the accuracy was determined to be around $1.5 \%$.

This calibration was then confirmed using a frequency method calibrating back through the subsystems of the detector to the piezo of the master laser. During lock, a signal was injected into one ESD. This induces an asymmetric length change in the Michelson cavity which gives rise to a length change in the power-recycling (PR) cavity. Varying the length of the PR cavity induces a change in the resonant frequency of the cavity. The frequency change that has to be imposed on the laser to keep the cavity on resonance gives a measure of the change in the cavity resonance frequency, and hence gives a measure of the length change of the PR cavity. This was done and the result for the IMD calibration agreed with the fringe counting method to within $15 \%$.

GEO has two electrostatic drives which provides the possibility to calibrate each drive while the detector is locked. This is done by locking the detector with only one ESD and then injecting a very low frequency signal into the other ESD. The induced displacement was measured by looking at the compensating signal seen in the feedback to the IMD of the same pendulum chain. As the calibration of the IMD was measured previously, it is possible to determine the calibration for this ESD. This experiment was repeated for the other ESD and the two calibration factors were consistent to within $1 \%$.

Although the response of the ESD is well known at low frequencies, it is also necessary to check the frequency response $\left(1 / f^{2}\right)$ of the ESD at high frequencies where interactions with the (then) steel suspension wires were thought to possibly alter the response.

During S1, the rms values of the calibration lines at 732, 1220 , and $1708 \mathrm{~Hz}$ were recorded at $1 \mathrm{~Hz}$. Using the assumed model of the ESD response, $1 / f^{2}$ from force to displacement, it is possible, for any of the recorded $1 \mathrm{~Hz}$ samples, to predict the value of any calibration peak based on the measured value of any of the other two. A long stretch of data was chosen $(24 \mathrm{~h})$ to investigate the validity of the ESD model. The resulting measurements of the ESD response differ from the $1 / f^{2}$ model by around $2.5 \%$, this is consistent with the errors introduced in the measuring process (signal-to-noise ratio of the calibration peaks) and so we conclude that the model is valid.

The frequency response of the interferometer (the optical gain) was assumed to be flat. This response is composed of a true optical part, followed by the response of the photodetector and the rf demodulation system. The main optical response is that of the interferometer arm, which has a flat frequency response below $6 \mathrm{kHz}$ (the first feature is a null at the inverse light travel time of the interferometer, around 125 $\mathrm{kHz}$ ). The photodiode and $\mathrm{rf}$ system bandwidth were of order $100 \mathrm{kHz}$, and were measured to have a flat frequency response below $6 \mathrm{kHz}$.

Since the optical gain is flat in the detection band, and (by design) there is no loop gain in the Michelson servo at the frequencies of the calibration lines, the confirmation of the ESD model is sufficient to validate the high-frequency calibration.

To confirm the low-frequency calibration we must look closely at the model of the Michelson servo loop. Measurements of the electronic transfer functions were used (with $\mathrm{LISO}^{3}$ ) to develop suitable IIR filters. The feedback actuators have already been discussed above, so the low frequency calibration depends only on a valid loop model.

Before the S1 run, measurements were made of the closed-loop transfer function of the Michelson servo. For the particular set of gains at the time of measurement, the model closed-loop transfer function calculated in the frequency domain was compared to the measurements. The optical gain was not measured at this time but an optical gain around the nominal value can be chosen to give the match shown in Fig. 7.

Equations (3) and (4) show the frequency domain representation of the model transfer function while Fig. 5 and Eq. (5) show a time-domain representation of the model. The differences between these two representations of the model (which can be attributed to the inability of time-domain IIR 

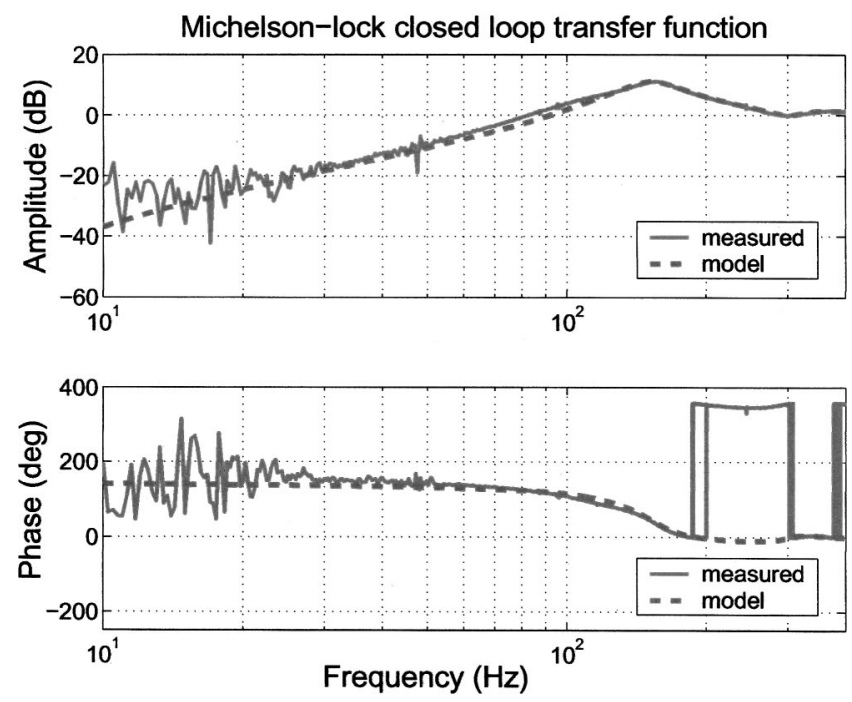

FIG. 7. Michelson lock closed loop transfer function measured and calculated for a particular set of gain parameters.

filters to perfectly match the analog equivalent), are shown in Fig. 8.

The confirmation that the model closely matches the measurements, and that a standard frequency-domain model can be closely approximated by the developed time-domain model, is the last link in the validation chain. The analysis of the calibration procedure over long time scales throws up some other interesting results. Figure 9 shows a highresolution amplitude spectra of the second calibration line. On the plot the calibration line is shown as it appears in both the recorded detector error-point and in the derived $h(t)$ signal. Each spectrum is the result of $1000 \mathrm{~s}$ of data split to give 50 spectral averages at $0.05 \mathrm{~Hz}$ resolution. A Hanning window was applied in the analysis. The two curves are lined up approximately by eye to show how they compare. From the plot it can be seen that the suppression of the $732 \mathrm{~Hz}$ peak is around $20 \mathrm{~dB}$ - enough so that the remaining peak does not dominate the numerical dynamic range of the signal. The
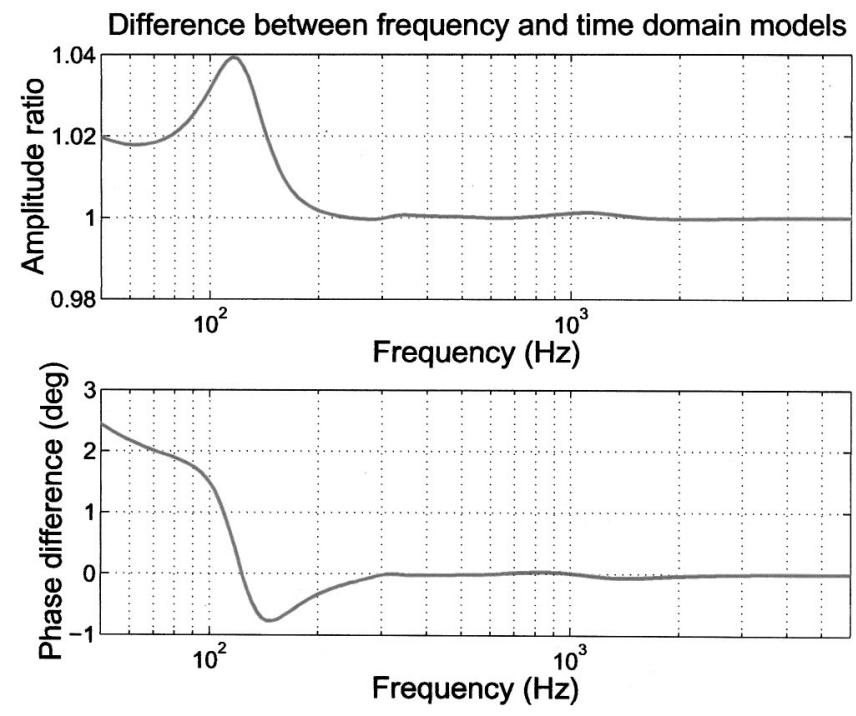

FIG. 8. The difference between the calibration function from $\mathrm{V}$ to $\mu \mathrm{m}$ calculated using the frequency- and time-domain models.

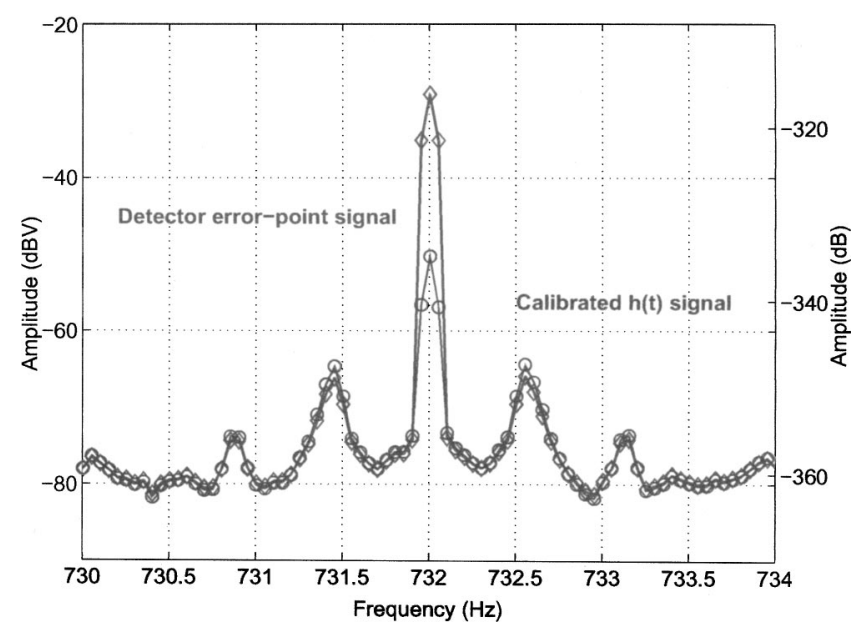

FIG. 9. The $732 \mathrm{~Hz}$ calibration line.

sidebands that appear in this plot show the presence of some amplitude modulation at about $0.6 \mathrm{~Hz}$. This observed frequency of the amplitude modulation is in good agreement with the dominant known residual pendulum motion provided by the systems which damp the pendulum modes. ${ }^{2}$ The fact that the modulation is still present in the calibrated $h(t)$ channel indicates that the gain variations were estimated too infrequently to correct for this amplitude modulation. Although this effect is not too significant, a move towards more frequent gain estimation would reduce the significance of this effect further.

\section{MODEL LIMITATIONS AND CALIBRATION ACCURACY}

The detector model has various limitations that are implicit to the design. The calibration of GEO is concentrated on the proposed $50 \mathrm{~Hz}-6 \mathrm{kHz}$ detection band of the instrument.

The lower limit of the calibration is defined as $10 \mathrm{~Hz}$. This is due to difficulties in designing an IIR filter that matches the transfer function of the pendulum responses which are thought to have the main pendulum resonance at around $0.6 \mathrm{~Hz}$. Since the sampling rate of the GEO DAQ system is $16384 \mathrm{~Hz}$, it is very difficult to design a stable IIR filter that has poles at $0.6 \mathrm{~Hz}$, so, to avoid any instabilities, the filters that map the pendulum responses are designed to have the resonance at $5 \mathrm{~Hz}$. A suitable gain factor is then applied to the filters so that the amplitude response above 10 $\mathrm{Hz}$ matches exactly that of a $0.6 \mathrm{~Hz}$ pendulum resonance. The phase response of the filter deviates from the analog equivalent at $10 \mathrm{~Hz}$ but converges quickly to give approximately the required response before reaching $50 \mathrm{~Hz}$.

The upper frequency limit of the calibration scheme is set by the hardware antialiasing filters present in the DAQ system to be about $6.5 \mathrm{kHz}$. It is difficult to correct for this using IIR filters as it is so close to the Nyquist frequency. In practise this has no significant consequences as no astronomical searches target these frequencies.

In the band $50 \mathrm{~Hz}$ to $\sim 6 \mathrm{kHz}$ the accuracy of the calibration process can best be examined in the low- and highfrequency regimes. At high frequencies the calibration accu- 
racy is dominated by our ability to measure the optical gain of the detector and is ultimately dominated by the calibration of the electrostatic drive. The method for determining the $\mathrm{dc}$ calibration of the ESD relies strongly on the accuracy of the calibration of the intermediate mass drive (IMD). For the S1 science run, the IMD was calibrated with an accuracy of about $1.5 \%$; the ESD was then calibrated with an accuracy of around $2.5 \%$ per drive.

At low frequencies, where the Michelson servo dominates, the calibration accuracy also depends on the accuracy with which we measure the relative gain of the two output photodiodes. This measurement is made from the calibration peaks in both the low- and high-power photodiode signals. The error in this measurement is dominated by the shot-noise of the low-power photodiode. Studies of the estimated variance of the relative gain measurements show that on time scales shorter than $1 \mathrm{~min}$ the error is about 3\%. In addition, we must consider the error introduced because of the differences between the time-domain filter responses and their analog equivalents (up to $4 \%$ ).

\section{FUTURE WORK}

Further calibration work will need to focus on the problems and deficiencies highlighted in this experiment. In addition, the introduction of the signal-recycling mirror into
GEO will require a more sophisticated calibration scheme. In particular the optical response of the detector will no longer be flat and will need to be tracked in a frequency dependent way. The gravitational wave signal will no longer be contained in a single demodulation quadrature of the output photodiode, so multiple output signals will need to be calibrated and combined to give a single calibrated strain channel.

In the future we aim to determine the calibration with a fractional error of about $1 \%$ by introducing a photon drive actuator into the calibration scheme. This photon drive actuator will use a laser at a different frequency to that of the main interferometer laser to apply a variable radiation force to the rear-side of one of the end mirrors; calibration of the photon-drive can be achieved with more accuracy than the calibration of the ESDs since the momentum transfer can be monitored with high accuracy by measuring the light reflected from the mirror. The use of the photon drive also allows continuous monitoring of the ESD calibrations.

\footnotetext{
${ }^{1}$ G. Heinzel, A. Freise, H. Grote, K. A. Strain, J. Hough, and K. Danzmann, Class. Quantum Grav. 19 (2002).

${ }^{2}$ M. V. Plissi, C. I. Torrie, M. E. Husman, N. A. Robertson, K. A. Strain, H. Ward, H. Lück, and J. Hough, Rev. Sci. Instrum. 71, 2539 (2000).

${ }^{3}$ G. Heinzel, Advanced Optical Techniques for Laser-Interferometric Gravitational-Wave Detectors (MPQ 243, 1999).

${ }^{4}$ Steven W. Smith, The Scientist and Engineer's Guide to Digital Signal Processing (Analog Devices).
} 